\title{
Camptothecin induces DNA strand breaks and is cytotoxic in stimulated normal lymphocytes
}

\author{
TAKAHIRO YAMAUCHI, AKIRA YOSHIDA and TAKANORI UEDA \\ Department of Hematology and Oncology, Faculty of Medical Sciences, University of Fukui, \\ 23-3 Shimoaizuki, Matsuoka, Eiheiji, Fukui 910-1193, Japan
}

Received September 13, 2010; Accepted October 27, 2010

DOI: $10.3892 /$ or.2010.1100

\begin{abstract}
Camptothecin (CPT), a topoisomerase I inhibitor, forms a cleavable complex with topoisomerase I and singlestranded DNA. When this complex meets a replication fork, the collision generates irreversible double-strand breaks, thereby inducing apoptosis. Based on the mechanism of action, we hypothesized that cycling cells would be more sensitive to CPT than non-cycling cells and that cells stimulated to undergo DNA synthesis would be sensitized to CPT. The study focused on the association between CPT-induced DNA strand breaks and apoptotic cell death, because the induction of DNA strand breaks is indispensable for cytotoxicity. We used the Comet assay to quantitate DNA strand breaks and Annexin $\mathrm{V}$ positivity to determine the level of cytotoxicity. Normal lymphocytes were used as a model for quiescent cells. First, the cultured leukemic cell line CCRFCEM was treated with CPT. CEM cells were sensitive to $\mathrm{CPT}$, and the amount of CPT-induced DNA strand breaks was concentration- and time-dependent. The increase in DNA strand breaks appeared to be correlated to a subsequent increase in apoptosis. When normal lymphocytes were treated with CPT, DNA strand breaks quickly disappeared, and the subsequent induction of apoptosis was minimal. However, when normal lymphocytes were stimulated to undergo DNA synthesis, the lymphocytes were sensitized to CPT with increased DNA strand breaks and enhanced apoptosis. Again, the extent of DNA strand breaks was associated with the magnitude of cytotoxicity. Thus, CPT was cytotoxic to stimulated normal lymphocytes in the context of DNA synthesis.
\end{abstract}

\section{Introduction}

Camptothecin (CPT) is a potent anticancer agent that was originally isolated from the bark of the Chinese tree

Correspondence to: Dr Takahiro Yamauchi, Department of Hematology and Oncology, Faculty of Medical Sciences, University of Fukui, 23-3 Shimoaizuki, Matsuoka, Eiheiji, Fukui 910-1193, Japan

E-mail: tyamauch@u-fukui.ac.jp

Key words: camptothecin, DNA strand break, Comet assay, quiescent cells
Camptotheca acuminata about 40 years ago $(1,2)$. Derivatives of CPT, including topotecan and irinotecan, have effectively been used to treat cancers of the colon, ovary, lung, pancreas, and esophagus and hematological malignancies (2-4). Thus, CPT has clinical utility in many cancer types, especially in relapsed or refractory situations.

The primary mechanism of action of CPT is the inhibition of human topoisomerase I (Topo I) (5). Relaxation of supercoiled DNA is a key function of Topo I. Topo I normally functions to relieve the torsional stress associated with DNA replication by inducing single-strand breaks in DNA (6-8). CPT reversibly forms the cleavable complex of CPT-Topo IDNA. When this complex meets a replication fork, the collision converts single-strand breaks into irreversible doublestrand breaks, thereby inducing apoptosis. Thus, based on the mechanism of action, the cytotoxicity of CPT is S-phase specific (9-13).

Because the level of Topo I is elevated in some cancers (14), Topo I poisons such as CPT are successfully employed in chemotherapy regimens. However, clinical resistance is common (9-12), suggesting the need for further improvements in CPT-based chemotherapy. The activity of CPT may be limited to the growth fraction of cancer, because the dormant subpopulation is thought to be insensitive to the drug. One strategy for enhancing the effect of CPT on cancer cells may be the induction of the cell cycle. Therefore, we hypothesized that cycling cells would be more sensitive to CPT than noncycling cells and that cells stimulated to undergo DNA synthesis would be sensitized to CPT.

In the present study, we used normal lymphocytes as an experimental model for the quiescent population of cancer cells. First, the cultured leukemic cell line CCRF-CEM was used to evaluate the effect of CPT in cycling cells. Because the induction of DNA strand breaks based on the collision theory is indispensable for the exertion of CPT's cytotoxicity, the study focused on the association between the amount of DNA strand breaks and the level of apoptotic cell death. Next, normal lymphocytes were used to assess the sensitivity of dormant cells to CPT. Lastly, the cytotoxicity of CPT was further determined in normal lymphocytes that were stimulated to undergo DNA synthesis.

\section{Materials and methods}

Chemicals and reagents. CPT was purchased from Sigma (St. Louis, MO, USA) and dissolved in 100\% dimethylsulfoxide. 
Cell culture. Human leukemia CCRF-CEM cells were cultured in RPMI-1640 supplemented with $10 \%$ heat-inactivated fetal calf serum. The cells were maintained in a $5 \% \mathrm{CO}_{2}$-humidified atmosphere at $37^{\circ} \mathrm{C}$.

Lymphocyte preparation. Normal lymphocytes from 4 healthy donors were used. Whole blood was drawn into heparinized tubes, layered over Ficoll-Hipaque solution, and centrifuged at $1500 \mathrm{rpm}$ for $30 \mathrm{~min}$. The lymphocytes were harvested from the interphase, washed twice with PBS and resuspended at a concentration of $1 \times 10^{6}$ cells $/ \mathrm{ml}$ in media. The cells were incubated in a $5 \% \mathrm{CO}_{2}$-humidified atmosphere at $37^{\circ} \mathrm{C}$ overnight. Lymphocytes were further incubated at $37^{\circ} \mathrm{C}$ with $500 \mathrm{ng} / \mathrm{ml}$ phytohemagglutinin-M (PHA) for $48 \mathrm{~h}$ for activation (15).

Drug treatment. CEM cells, activated normal lymphocytes, or inactivated normal lymphocytes $\left(5 \times 10^{5}\right.$ cells $\left./ \mathrm{ml}\right)$ were incubated with various concentrations of CPT for the indicated time periods. Then, cells were washed and incubated in fresh media for the indicated time periods. The cells were then evaluated for growth, viability and the induction of DNA strand breaks.

The alkaline single cell gel electrophoresis (Comet) assay. To quantitate the amount of DNA strand breaks, the alkaline Comet assay was performed according to the previously described method (16-18). After treatment with CPT, the cells were mixed with $20 \mu \mathrm{l}$ of $0.5 \%$ low melting point agarose in PBS at $37^{\circ} \mathrm{C}$. The mixture was layered onto a frosted microscope slide previously coated with $70 \mu 1$ of $0.65 \%$ normal agarose in PBS, followed by a top layer of $80 \mu 1$ of low melting point agarose. After solidification, the slide was left in lysis solution $(2.5 \mathrm{M} \mathrm{NaCl}, 10 \mathrm{mM}$ Tris, $100 \mathrm{mM}$ ethylenediamine tetraacetic acid, $10 \%$ dimethylsulfoxide, $1 \%$ Triton $\mathrm{X}-100, \mathrm{pH} 10)$ at $4^{\circ} \mathrm{C}$ for at least $1 \mathrm{~h}$. The slide was then placed in the electrophoretic buffer $(1 \mathrm{mM}$ ethylenediamine tetraacetic acid, $300 \mathrm{mM} \mathrm{NaOH}, \mathrm{pH}$ 13) to allow unwinding of DNA, followed by electrophoresis. After electrophoresis, the slide was washed, neutralized and stained with ethidium bromide. One hundred cells per treatment were analyzed by using a computer-based image analysis system (Kinetic Imaging Komet system, Ver. 4.0, Liverpool, UK). The amount of DNA strand breaks was expressed as the 'tail moment', which is a combined measurement of the length of the DNA migration and the relative DNA content.

Evaluation of DNA synthesis. To evaluate the activation of normal lymphocytes, the initiation of DNA synthesis was determined as an incorporation of tritiated thymidine into the nuclear DNA (16). In brief, normal lymphocytes ( $2 \times 10^{6}$ cells) that had been previously treated with PHA for $48 \mathrm{~h}$ were washed in fresh media and incubated with tritiated thymidine ( $3 \mu \mathrm{Ci} / \mathrm{ml}$ ) for the indicated time periods. The lymphocytes were then collected by centrifugation ( $1500 \mathrm{rpm}, 5 \mathrm{~min})$ and resuspended in $500 \mu 1$ of $0.4 \mathrm{~N}$ perchloric acid. The sample was mixed, centrifuged and resuspended in perchloric acid. After another mixing and centrifugation, the pellet was resuspended in $1 \mathrm{ml}$ of $0.5 \mathrm{~N} \mathrm{KOH}$ and incubated at $45^{\circ} \mathrm{C}$ over- night to dissolve the pellet. Radioactivity (per $2 \times 10^{6}$ cells) was counted on the following day.

Proliferation assay. To evaluate the cell proliferation, a sodium 3'-\{1-[(phenylamino)-carbonyl-3,4-tetrazolium]\}-bis(4methoxy-6-nitro) benzene sulfonic acid hydrate (XTT) assay was performed according to the manufacturer's instructions (Roche, Indianapolis, IN, USA) with slight modifications (19). In brief, $1 \mathrm{ml}$ of a cell suspension $\left(5 \times 10^{4} / \mathrm{ml}\right)$ was incubated for $24 \mathrm{~h}$ in a 24 -well plate, followed by the addition of a 10- $\mu 1$ aliquot of CPT solution at various concentrations. The cells were incubated with CPT for $72 \mathrm{~h}$, and a $100-\mu 1$ aliquot was transferred to a $96-$ well microplate. The cells were then mixed with $50 \mu \mathrm{l}$ XTT and incubated for $4 \mathrm{~h}$ at $37^{\circ} \mathrm{C}$. The samples were applied to a spectrophotometer, and the $\mathrm{IC}_{50}$ value was extrapolated from a growth inhibition curve.

Quantitation of apoptotic cell death. To evaluate the cytotoxicity of CPT, apoptotic cell death was determined by phosphatidylserine externalization at 4 or $24 \mathrm{~h}$ after treatment (16). CPT-treated cells were collected by centrifugation (1500 rpm, $5 \mathrm{~min}$ ) and resuspended in $200 \mu \mathrm{l}$ of binding buffer $(10 \mathrm{mM}$ HEPES/NaOH, pH 7.4, $140 \mathrm{mM} \mathrm{NaCl}$ and $2.5 \mathrm{mM} \mathrm{CaCl}_{2}$ ) and $10 \mu \mathrm{l}$ of FITC-conjugated Annexin V. The sample was incubated for $15 \mathrm{~min}$ at room temperature and centrifuged again. The pellet was resuspended with $500 \mu \mathrm{l}$ of the binding buffer and with $10 \mu \mathrm{l}$ of $50 \mu \mathrm{g} / \mathrm{ml}$ propidium iodide. The samples were analysed with a FACScan flow cytometer (Becton-Dickinson, San Jose, CA, USA). Data acquisition and analysis were performed by the CellQuest program (Becton-Dickinson). Cells positive for Annexin V were considered apoptotic (16).

Statistical analyses. All statistical analyses were performed with Microsoft Excel 2007 software (Microsoft, Redmond, WA, USA). All graphs, linear regression lines and curves were generated by using GraphPad Prism software (version 5.0) (GraphPad Software, Inc. San Diego, CA, USA). P-values $\leq 0.05$ were considered statistically significant.

\section{Results}

DNA strand breaks induced by CPT in CEM cells. The creation of DNA strand breaks by the collision between the CPT-Topo I-DNA cleavable complex and a replication fork is a critical event prior to the exertion of CPT-mediated cytotoxicity $(1,2)$. The Comet assay was used to measure DNA strand breaks in cycling cultured leukemic cells. When CEM cells were incubated with CPT, the tail moment values increased in a concentration- and time-dependent manner (Fig. 1A and B), indicating that DNA strand breaks were produced by CPT. When CEM cells were pulsed with $1 \mu \mathrm{M}$ CPT and then washed in fresh media, the tail moment was the highest at the end of the treatment and then decreased with time, returning to the control level at $4 \mathrm{~h}$ (Fig. 1C and D). This result suggests a loss of the drug effect that allowed rapid rejoining of DNA strand breaks. However, when the cells were treated with a higher concentration of CPT $(5 \mu \mathrm{M})$, the tail moment was retained with time, suggesting the 

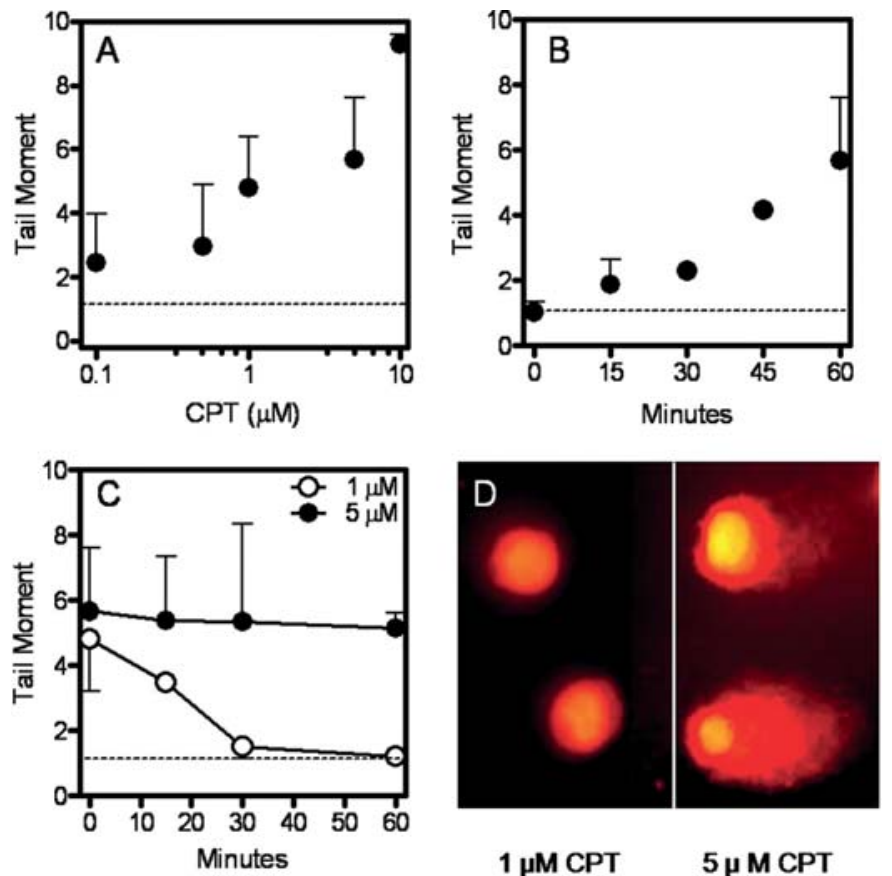

Figure 1. CEM cells were incubated with the indicated concentrations $(0.1,0.5,1,5$ and $10 \mu \mathrm{M}$ ) of CPT for $1 \mathrm{~h}$ (A) or incubated with $5 \mu \mathrm{M}$ CPT for the indicated time periods (B). After washing in fresh media, the cells were immediately applied to the Comet assay to measure DNA strand breaks. (C) Time course of DNA strand breaks produced by CPT. CEM cells were pulsed for $1 \mathrm{~h}$ with $1 \mu \mathrm{M}$ (०) or $5 \mu \mathrm{M}(\bullet)$ of CPT, followed by washing in fresh media and subsequent incubation. The cells were then applied to the Comet assay at $0 \mathrm{~h}, 15,30$ and $60 \mathrm{~min}$ after washing. (D) Typical comet images of CEM cells at $1 \mathrm{~h}$ after a pulse treatment with 1 or $5 \mu \mathrm{M} \mathrm{CPT}$ and subsequent washing in fresh media. The tail moment value of untreated cells was set as a control (dotted line). The values are the means \pm SD of triplicate determinations.
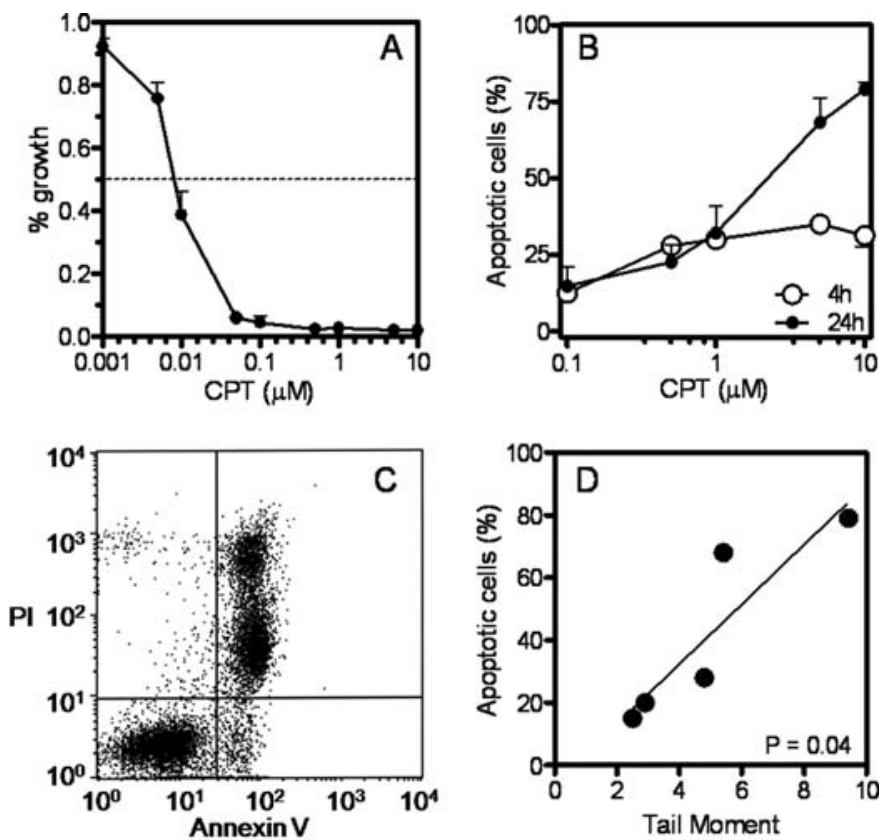

Figure 2. (A) Growth inhibition of CEM cells by CPT. CEM cells were incubated for $72 \mathrm{~h}$ with various concentrations of CPT and applied to the XTT assay. (B) CPT-mediated apoptotic cell death. CEM cells were incubated with the indicated concentrations $(0.1,0.5,1,5$ and $10 \mu \mathrm{M})$ of CPT for $1 \mathrm{~h}$, followed by washing and resuspension in fresh media. The cells were subsequently incubated and evaluated for Annexin V positivity after $4 \mathrm{~h} \mathrm{( \circ )} \mathrm{or} 24 \mathrm{~h}(\bullet)$. The values are the means \pm SD of triplicate determinations. (C) Representative flow cytometric image of CEM cells treated with $5 \mu$ M CPT. (D) Relationship between the amount of DNA strand breaks and the apoptotic cell death induced by the CPT treatment. The values determined above were plotted at each corresponding concentration of CPT. $\mathrm{R}^{2}=0.80, \mathrm{P}=0.04$.

persistence of DNA strand breaks without rejoining (Fig. 1C and D). These results suggest that CPT-induced DNA strand breaks reflect the intracellular action of CPT.
Cytotoxicity of CPT in CEM cells. The cytotoxicity of CPT was evaluated in terms of both the growth inhibition and the induction of apoptosis. The XTT assay revealed that the $\mathrm{IC}_{50}$ 


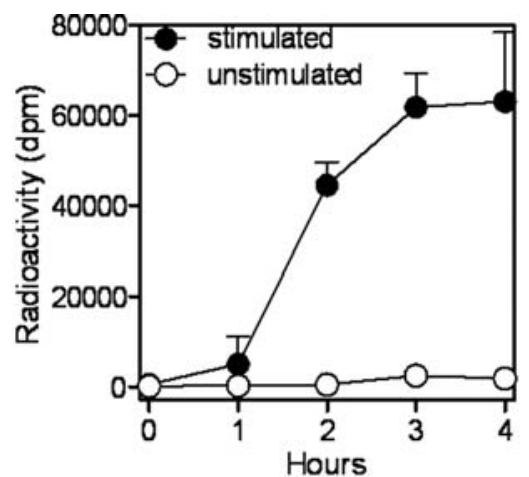

Figure 3. Normal lymphocytes $\left(1 \times 10^{6} / \mathrm{ml}\right)$ were stimulated to undergo DNA synthesis by incubation with (•) or without (०) $500 \mathrm{ng} / \mathrm{ml}$ PHA for $48 \mathrm{~h}$, followed by incubation with tritiated thymidine for the indicated time periods. The radioactivity of DNA was determined by scintillation counting. The values are the means \pm SD of triplicate determinations.

value after a 72-h incubation with CPT was $10 \mathrm{nM}$ for CEM (Fig. 2A), suggesting that the cells were quite sensitive to CPT. Flow cytometry demonstrated that a 24-h CPT treatment, but not a 4-h treatment, induced apoptotic cell death in a concentration-dependent manner (Fig. 2B and C). Importantly, the apoptotic cell death was proportional to the tail moment value generated by $\mathrm{CPT}$ at the corresponding concentration (Fig. 2D). These results suggest that the induction of DNA strand breaks is critical to the cytotoxicity of CPT.

Activation of normal lymphocytes. DNA synthesis is a marker for the activation of normal lymphocytes. The initiation of DNA synthesis was clearly demonstrated as the uptake of tritiated thymidine into DNA in PHA-stimulated normal lymphocytes (Fig. 3).

DNA strand breaks induced by CPT in normal lymphocytes. Because CPT-induced DNA strand breaks appeared to be directly associated with the exertion of cytotoxicity (Fig. 2D), the amount of DNA strand breaks was compared between stimulated and unstimulated lymphocytes. When quiescent lymphocytes were incubated for $4 \mathrm{~h}$ with CPT followed by
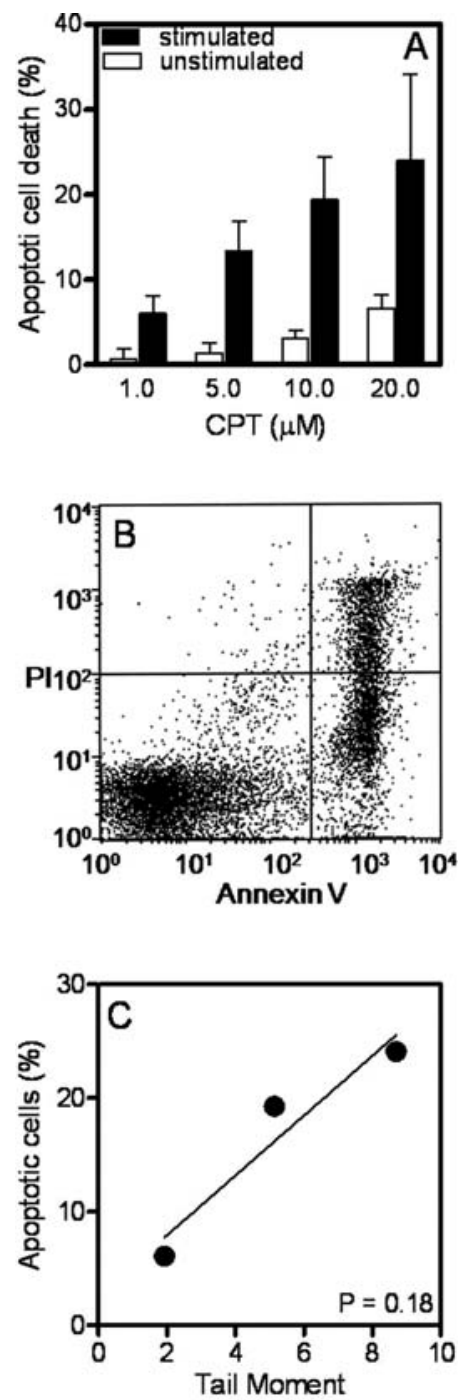

Figure 5. Normal lymphocytes were incubated for $48 \mathrm{~h}$ with $500 \mathrm{ng} / \mathrm{ml} \mathrm{PHA}$ (to stimulate DNA synthesis) or without PHA. The cells were subsequently incubated with the indicated concentrations of CPT for $4 \mathrm{~h}$, followed by washing and resuspension in fresh media. Apoptotic cell death was determined as Annexin V positivity after $24 \mathrm{~h}(\mathrm{~A})$. The values are the means \pm SD of triplicate determinations. (B) Representative flow cytometric image of cells treated with $20 \mu \mathrm{M}$ CPT. (C) Relationship between the amount of DNA strand breaks and the induction of apoptosis by CPT. Values were plotted at each corresponding concentration of CPT. $\mathrm{R}^{2}=0.92, \mathrm{P}=0.18$.
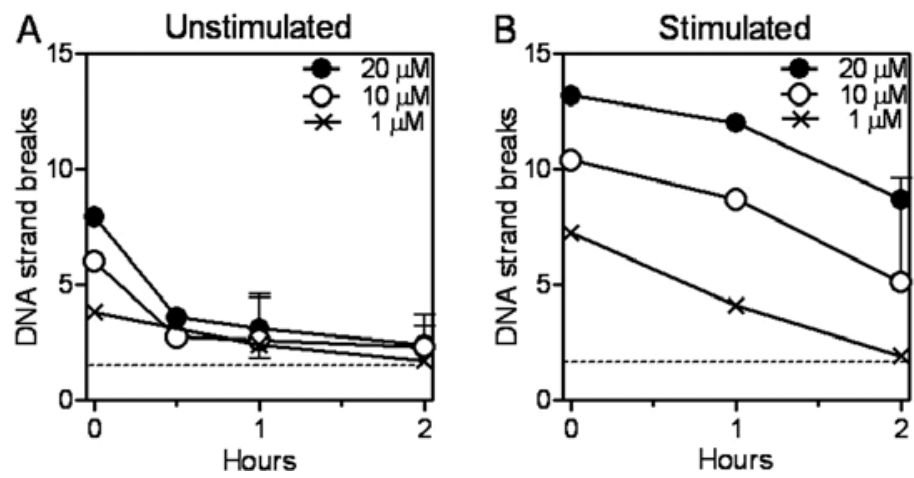

Figure 4. Normal lymphocytes that had (B) or had not (A) been stimulated with $500 \mathrm{ng} / \mathrm{ml}$ PHA for $48 \mathrm{~h}$ were incubated with various concentrations (1 $\mu \mathrm{M}$ (x), $10 \mu \mathrm{M}(\mathrm{O}), 20 \mu \mathrm{M}(\bullet)$ of CPT for $4 \mathrm{~h}$, followed by washing and resuspension in fresh media. The cells were subsequently incubated, and DNA strand breaks were quantitated at the indicated time-points by using the Comet assay. The tail moment value of untreated cells was set as a control (dotted line). The values are the means $\pm \mathrm{SD}$ of triplicate determinations. 
washing and incubation in fresh media, only a few DNA strand breaks were generated, and they disappeared within $2 \mathrm{~h}$ regardless of the drug concentration (Fig. 4A). To the contrary, DNA strand breaks were greater and more persistent in stimulated lymphocytes than in unstimulated lymphocytes (Fig. 4B). The results suggest that stimulated lymphocytes are more vulnerable to $\mathrm{CPT}$.

Cytotoxicity of CPT in normal lymphocytes. We hypothesized that CPT might be ineffective in quiescent normal lymphocytes but able to induce DNA strand breaks and mediate apoptosis in stimulated lymphocytes. To determine if CPT was cytotoxic to normal lymphocytes, the positivity of Annexin V was quantitated (Fig. 5) $24 \mathrm{~h}$ after lymphocytes were treated with CPT. A concentration of $20 \mu \mathrm{M}$ CPT was minimally toxic to non-cycling lymphocytes but successfully induced apoptosis in stimulated lymphocytes (Fig. 5A). The results clearly indicate that CPT was active in cycling cells but not in quiescent cells. Moreover, the cytotoxicity was augmented with increasing concentrations of CPT (Fig. 5A), and the apoptotic cell death was proportional to the 2-h tail moment value generated by CPT at the corresponding concentration (Fig. 5C). The results suggest that the initiation of DNA synthesis sensitized normal lymphocytes to CPT.

\section{Discussion}

A number of CPT-resistant cell lines have been isolated and characterized, revealing three categories of CPT resistance (10-13). In the first category, resistance is related to alterations in cellular drug accumulation/distribution and metabolism. Cellular efflux of the drug by P-glycoprotein or multidrug resistance protein and hepatic metabolism of the drug may have some contribution to the resistance. In the second category, resistance is caused by alterations in Topo I. A reduction in Topo I cleavable complexes can be caused by Topo I mutations that render the enzyme drug-resistant or that decrease the enzyme activity. In the third category, resistance is caused by factors downstream from cleavable complex resolution. These factors include alterations in DNA repair, cell cycle checkpoint and the apoptotic pathway. Thus, several mechanisms of drug resistance have been implicated. However, CPT is an S-phase-specific agent that damages the replication fork, and the critical point is that CPT is active only in cycling cells.

The present study was conducted to evaluate the cytotoxicity of CPT in stimulated lymphocytes in comparison with quiescent lymphocytes. First, we confirmed that the cycling cultured leukemic cell line CEM was sensitive to CPT and that its cytotoxic effect was concentration- and time-dependent (Fig. 1). A high concentration of CPT generated durable DNA strand breaks, thereby inducing apoptosis (Fig. 1). The extent of DNA strand breaks produced by CPT appeared to be correlated to an increase in apoptotic events (Fig. 2). Next, the effect of CPT was evaluated in quiescent normal lymphocytes. CPT-induced DNA strand breaks disappeared quickly, and the subsequent induction of apoptosis was minimal (Figs. 4 and 5). Lastly, normal lymphocytes were stimulated to undergo DNA synthesis (Fig. 3). Although CPT was not cytotoxic to quiescent lympho- cytes, PHA sensitized lymphocytes to CPT (Figs. 4 and 5). Again, the extent of DNA strand breaks was associated with the magnitude of the subsequent cytotoxicity (Fig. 5). Thus, the present study confirmed that CPT was cytotoxic to cycling cells in the context of DNA synthesis.

The Comet assay was successfully used for evaluating the action of CPT. CPT mediates cytotoxicity by binding reversibly to the Topo I - DNA complex $(1,2,11-13)$. The collision between this ternary complex and a replication fork generates irreversible DNA strand breaks, thereby inducing apoptotic cell death. The concentration- and time-dependent increase in the tail moment value (Figs. 1 and 2) suggests that DNA strand breaks were generated in response to CPT. The close association between the generation of DNA strand breaks and the induction of apoptosis suggests that collisionmediated irreversible DNA strand breaks lead to cytotoxicity (Fig. 2). When unstimulated normal lymphocytes were treated with CPT, DNA strand breaks were not durable, and CPT did not induce apoptosis. This suggested that the CPT-induced cleavable complex was reversible and did not encounter a replication fork that was indispensable to the exertion of the cytotoxicity. In PHA-stimulated lymphocytes, DNA strand breaks became greater and more persistent than in quiescent lymphocytes. This observation suggests that the initiation of DNA synthesis mediated the collision between the cleavable complex and a replication fork that caused irreversible DNA strand breaks. CPT at $20 \mu \mathrm{M}$, a concentration that was minimally toxic to unstimulated lymphocytes, caused apoptosis in activated lymphocytes, suggesting the necessity of DNA synthesis for the cell cycle-dependent activity of CPT. Thus, the amount of DNA strand breaks reflected the kinetics of the intracellular action of CPT, which was successfully quantitated by using the Comet assay.

The effect of CPT was previously evaluated in the context of quiescent lymphocytes by Bruno et al $(20,21)$. They demonstrated that the sensitivity of lymphocytes to CPT varied markedly depending on the stage of mitogenic stimulation. It was shown that quiescent $\mathrm{G}_{0}$ lymphocytes were almost insensitive to the drug, while CPT was quite cytotoxic to cells progressing through $\mathrm{S}$ and $\mathrm{G}_{2}$. These results were in accordance with the present findings, indicating cell cyclespecific activity of CPT. However, in light of the mechanisms of action, the kinetics of DNA strand breaks would more precisely reflect the intracellular process after the insult of CPT than the cell-cycle analysis. Thus, the present study has clearly demonstrated that CPT was active in normal lymphocytes undergoing DNA synthesis and that the cytotoxic activity of CPT was correlated with DNA strand breaks.

In conclusion, we demonstrated that stimulated normal lymphocytes were sensitive to CPT and that DNA strand breaks were closely associated with the cytotoxicity. Although quiescent cells are insensitive to cell cycle-specific agents including CPT, manipulations that can induce tumor cells to initiate DNA synthesis may provide new therapeutic strategies for enhancing the clinical efficacy of CPT.

\section{Acknowledgements}

This study was supported in part by a Grant-in-Aid from the Ministry of Education, Science and Culture of Japan (2007) 
and a Grant from the Japanese Society of Clinical Pharmacology and Therapeutics (2007).

\section{References}

1. Pommier Y: DNA topoisomerase I inhibitors: chemistry, biology and interfacial inhibition. Chem Rev 109: 2894-2902, 2009.

2. Takimoto $\mathrm{CH}$ and Arbuck SG: Topoisomearse I targeting agents: the camptothecins. In: Cancer Chemotherapy and Biotherapy. 3rd edition. Chabner BA and Longo DL (eds). Lippincott-Raven Publishers, Philadelphia, pp579-646, 2001.

3. Saltz LB, Cox JV, Blanke C, et al: Irinotecan plus fluorouracil and leucovorin for metastatic colorectal cancer. Irinotecan Study Group. N Engl J Med 343: 905-914, 2000.

4. Venditto VJ and Simanek EE: Cancer therapies utilizing the camptothecins: a review of the in vivo literature. Mol Pharmaceut 7: 307-349, 2010.

5. Pommier Y: Topoisomerase I inhibitors: camptothecins and beyond. Nat Rev Cancer 6: 789-802, 2006.

6. Pizzolato JF and Saltz LB: The camptothecins. Lancet 361: 2235-2242, 2003.

7. Thomas CJ, Rahier NJ and Hecht SM: Camptothecin: current perspectives. Bioorg Med Chem 12: 1585-1604, 2004.

8. Schneider E, Hsiang YH and Liu LF: DNA topoisomerases as anticancer drug targets. Adv Pharmacol 21: 149-183, 1990.

9. $\mathrm{Xu} \mathrm{Y}$ and Villalona-Calero MA: Irinotecan: mechanisms of tumour resistance and novel strategies for modulating its activity. Ann Oncol 13: 1841-1851, 2002.

10. Pommier Y, Gupta M, Valenti M and Nieves-Neira W: Cellular resistance to camptothecins. Ann NY Acad Sci 803: 60-73, 1996.

11. Pommier Y, Pourquier P, Fan Y and Strumberg D: Mechanism of action of eukaryotic DNA topoisomerase I and drugs targeted to the enzyme. Biochim Biophys Acta 1400: 83-105, 1998.
12. Pommier $\mathrm{Y}$, Pourquier $\mathrm{P}$, Urasaki $\mathrm{Y}, \mathrm{Wu} \mathrm{J}$ and Laco GS: Topoisomerase I inhibitors: selectivity and cellular resistance. Drug Resist Updat 2: 307-318, 1999.

13. Urasaki Y, Takebayashi Y and Pommier Y: Activity of a novel camptothecin analogue, homocamptothecin, in camptothecinresistant cell lines with topoisomerase I alterations. Cancer Res 60: 6577-6580, 2000

14. Kaufmann S, Charron M, Burke PJ and Karp JE: Changes in topoisomerase I levels and localisation during myeloid maturation in vitro and in vivo. Cancer Res 55: 1255-1260, 1995.

15. Lichtman AH, Segel GB and Lichtman MA: The role of calcium in lymphocyte proliferation. Blood 61: 413-422, 1983.

16. Yamauchi T, Nowak BJ, Keating MJ and Plunkett W: DNA repair initiated in chronic lymphocytic leukemia lymphocytes by 4-hydroperoxycyclophosphamide is inhibited by fludarabine and clofarabine. Clin Cancer Res 7: 3580-3589, 2001.

17. Yamauchi T, Kawai Y and Ueda T: 1-B-D-arabinofuranosylcytosine is cytotoxic in quiescent normal lymphocytes undergoing DNA excision repair. Cancer Sci 93: 1334-1341, 2002.

18. Yamauchi T, Ogawa M and Ueda T: Carmustine-resistant cancer cells are sensitized to temozolomide as a result of enhanced mismatch repair during the development of carmustine resistance. Mol Pharmacol 74: 82-91, 2007.

19. Yamauchi T, Nishi R, Kitazumi K, Nakano T and Ueda T: A new high-performance liquid chromatography method determines low production of $9-\beta-D$-arabinofuranosylguanine triphosphate, an active metabolite of nelarabine, in adult T-cell leukemia cells. Oncol Rep 23: 499-504, 2010.

20. Bruno S, Giaretti W and Darzynkiewicz Z: Effect of camptothecin on mitogenic stimulation of human lymphocytes: involvement of DNA topoisomerase I in cell transition from G0 to G1 phase of the cell cycle and in DNA replication. J Cell Physiol 151: 478-486, 1992.

21. Gallo RC, Whang-Peng J and Adamson RH: Studies on the antitumor activity, mechanism of action, and cell cycle effects of camptothecin. J Natl Cancer Inst 46: 789-795, 1971. 